\title{
Efficient Data Collection from Wireless Nodes under the Two-Ring Communication Model
}

\author{
Onur Tekdas Deepak Bhadauria Volkan Isler *
}

January 26, 2012

\begin{abstract}
We introduce a new geometric robot routing problem which arises in data muling applications where a mobile robot is charged with collecting data from stationary sensors. The objective is to compute the robot's trajectory and download sequence so as to minimize the time to collect the data from all sensors. The total data collection time has two components: the robot's travel time and the download time. The time to download data from a sensor $s$ is a function of the locations of the robot and $s$ : If the robot is a distance $r_{i n}$ away from $s$, it can download the sensor's data in $T_{i n}$ units of time. If the distance is greater than $r_{i n}$ but less than $r_{\text {out }}$, the download time is $T_{\text {out }}>T_{\text {in }}$. Otherwise, the robot can not download the data from $s$. Here, $r_{\text {in }}, r_{\text {out }}, T_{\text {in }}$ and $T_{\text {out }}$ are input parameters. We refer to this model, which is based on recently developed experimental models for sensor network deployments, as the two ring model, and the problem of downloading data from a given set of sensors in minimum amount of time under this model as the Two-Ring Tour (TRT) problem.

We present approximation algorithms for the general case which uses solutions to the Traveling Salesperson with Neighborhoods (TSPN) Problem as subroutines. We also present efficient solutions to special but practically important versions of the problem such as grid-based and sparse deployments. The approach is validated in outdoor experiments.
\end{abstract}

\section{Introduction}

Research on Wireless Sensor Networks (WSNs) has been very active in the last two decades with researchers focusing on issues ranging from deployments with coverage guarantees [22] to the development of energy-efficient communication protocols to improve network lifetime [1]. Consequently, the technology advanced to the level that WSNs are now being routinely used in environmental applications such as the monitoring of humidity levels to determine vineyard irrigation levels [26]. In fact, commercial products are now available for building such systems.

Using mobility to improve ad-hoc networks $[21,36,12]$ and WSNs is studied extensively in the literature. In WSNs mobile entities can be used as data mules to collect data from sensor nodes. From wireless communications perspective using robots as data mules has a number of advantages over stationary WSNs: robots eliminate the need for deploying relay nodes to collect data. This may drastically reduce the number of necessary nodes in large scale habitat monitoring applications. In addition, since the robot can move close to the sensing node, the energy consumed by the nodes for communication is decreased which, in turn, significantly improves the network's lifetime $[29,31]$.

${ }^{*}$ The authors are with the Department of Computer Science and Engineering at the University of Minnesota, 200 Union St SE, Minneapolis, MN 55455, USA. Emails: \{bhadau,isler, tekdas\}@cs.umn.edu. This work is supported by NSF Awards \#0916209, \#0917676, \#0936710 and a fellowship from the Institute on the Environment at the University of Minnesota. 
Most of the problems in this context are studied under the assumption that the data mule is an uncontrolled entity. In [27], the authors present the details of a three tier sensor network architecture. The real implementation of similar systems are presented in $[19,6]$. If the mobility model of the data mules is known before hand, this knowledge can be used to improve energy consumption of the sensor nodes. Energy consumption can be reduced by using efficient protocols and scheduling algorithms for the wireless sensor nodes [9, 18, 8, 3, 2]. A recent review on the state of the art in exploiting sink mobility can be found in $[15,17,33]$

If the data mules is a controlled entity such as a robot, planning its path becomes a fundamental problem: Given the location of wireless devices, find the robot's path (and a download schedule) which minimizes the time to collect data from all nodes. If the download times are identical and we force the robot to travel to the exact locations of the sensors, then the problem is identical to the Traveling Salesperson Problem (TSP). However, traveling to the exact location of each device is not necessary. If we assume that the time to download the data of sensor node $s$ is uniform inside a disk centered at $s$, the problem becomes similar to the geometric version of TSP with Neighborhoods (TSPN): given $n$ uniform disks on the plane, compute the shortest path that touches all the disks $[4,13]$. In multi-robot data-muling applications, rather than simply minimizing the tour length for each robot, the time spent in traveling and downloading must be minimized. In our recent work, we presented an algorithm which balances the load shared by the robots by minimizing the maximum time spent by the robots for data gathering [7].

Our recent field work [7] revealed that the uniform disk model does not always accurately capture the time spent in downloading a fixed amount of data in data muling applications. In general, modeling packet delivery performance is a difficult problem as the quality of wireless communication depends on many parameters such as "the environment, the part of the frequency spectrum under use, the particular modulation schemes under use and possibly on the communication devices themselves [35]." However, recent results in the WSN community [35, 10] show that while the signal strength is a continuously decaying function, packet loss exhibits a step behaviour in existing WSNs (cf. Figures 10 and 12 in [35]). This suggests that the following two ring communication model models the dependency of communication quality on distance accurately in most sensor network deployments. In this model, there are two concentric disks centered at the sensor location. Inside the inner disk the communication is reliable thus the expected download time is shorter. Between the boundaries of the inner and the outer disks, communication is possible however due to increase in packet loss rate, the expected download time increases.

Collecting data under two-ring communication model is a new optimization problem which we call Two-Ring Tour Problem (TRT). In this version of the problem the tours are no longer optimal TSPN paths visiting uniform disks. An optimal tour is the one which trades-off between downloading from the outer disk or going further to download from the inner disk with shorter download time (Figure 1).

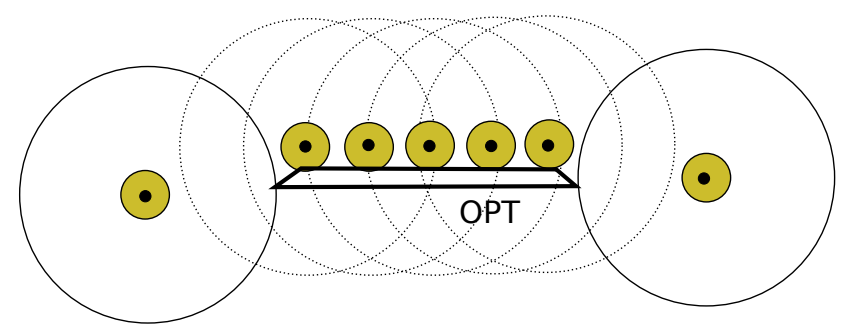

Figure 1: A TRT instance. Each sensor has a two ring communication model. If the robot enters the inner disk (shaded region), it downloads data faster than downloading from the outer disk. For this instance the optimal solution visits a mixture of inner and outer disks.

Our Contributions: We first study the general case of collecting data from sensors on the plane. In Section 3.1, we present a $(p+q)$-approximation where $p$ and $q$ are the approximation 
factors for TSPN and k-TSPN problems (These problems will be defined shortly). Next we present easy to implement polynomial algorithms for TRT which have approximation factors $O\left(T_{\text {out }} / T_{\text {in }}\right)$ and $O\left(r_{\text {out }} / r_{\text {in }}\right)$ (Section 3.2 and Section 3.3).

We also study the following special cases for TRT problem: non-intersecting outer disks and grid-based deployments both of which occur frequently in practical applications. When the outer disks are non-intersecting, we present a constant factor approximation algorithm (Section 4.1) based on a novel lower bound presented in Theorem 1. When sensors are deployed uniformly on a grid (with possibly intersecting disks), we formalize the problem as an integer linear program, solve the relaxed version (linear program) optimally and show how it can be rounded to obtain a 4-approximation (Section 4.2). In Section 5, we present results from a field experiment performed using a ground robot.

We start the paper with an overview of related work on data mules (Section 1.1), and TSP and TSPN problems (Section 1.2), and give a formal definition of the TRT problem in Section 1.3.

\subsection{Related Work on Data Mules}

Using data mules as controllable agents has recently received the attention of the researchers in the networking community. In [20] the authors consider the control of the robot's velocity along a fixed path to improve transmission quality. Similarly [28] study the speed control problem for the data mule when it is downloading data from a sensor while traveling within its communication range. In both works, the authors do not address the path planning problem.

In [30], the authors present heuristics to improve data latency and data aggregation rate in a data mule system where mobile agent is responsible for collecting data from cluster heads. [32] presents a heuristic for minimizing path length of a data mule to collect data from spatially separated WSNs. In our work we focus on the travel time of the mobile agents and we present algorithms with theoretical performance guarantees. In our previous work [29], we implemented a system which uses TSP and k-TSP algorithms to find efficient strategies for multiple data mules. A similar system is designed for an underwater data muling system in [14]. In [34], the authors formulate the problem of collecting sensor data using a single robot as an instance of the TSP with neighborhoods (TSPN) problem. In [7], we presented multi-robot algorithms which also used the disk model but took both the distance traveled and download time into consideration. In all of the above work, the communication range is either modeled as a single point or a disk. In our present work we use a realistic communication model (the two-ring model) and address the tour time which incorporates both the traveling times and the download times.

\subsection{Related Work on TSPN and Variants}

The Traveling Salesman Problem (TSP) is one of the most widely studied combinatorial optimization problems. Even though TSP in its general form is inapproximable, the metric version admits a constant factor approximation [11]. The Euclidean version in any fixed dimension admits a Polynomial Time Approximation Scheme (PTAS) [24, 5]. A generalization of TSP which received significant recent attention is TSP with Neighborhoods (TSPN) [4, 13]. In a TSPN instance, instead of $n$ points we are given $n$ neighborhoods (a neighborhood is a bounded region). The goal is to find a minimum cost tour which visits at least one point in each neighborhood.

Arkin and Hassin [4] introduced TSPN and presented a $(3 \sqrt{2}+1) p$ approximation algorithm for the case when neighborhoods are equal length parallel segments (Here, $p$ is the approximation ratio for TSP). For neighborhoods which are translates of a convex region, they gave a $\left(\sqrt{7^{2}+3^{2}}+1\right) p$ approximation algorithm.

The version of TSPN where the neighborhoods are uniform disks has many applications. In fact, the TRT problem, introduced here, is a generalization of this problem which in turn generalizes Euclidean TSP.

Dumitrescu and Mitchell presented constant factor approximation algorithms for TSPN with uniform disks [13]. In this work, they start with the case where the disks are disjoint. They show that a TSP tour that visits the centers of the disks is an $O(1)$-approximation to the TSPN 
tour. In Section 2, we present a novel lower bound for TSPN with uniform disks which is of independent interest and is used in the analysis of algorithms presented in Section 3.3 and Section 4.1.

For the general case where the disks can be intersecting, Dumitrescu and Mitchell first compute a maximal independent set $I$ of the input set (in other words, they compute a maximal set of non-intersecting disks). They form a tour which visits the centers of disks in $I$, and traverses their perimeters (to visit disks not included in $I$ ). It is shown that the length of this tour is within a constant factor of the optimal TSPN tour. In Section 3.3, we use this strategy of computing an independent set and visiting these disks as a subroutine to compute a TRT tour.

In more recent work, Mitchell showed that when the disks are disjoint, TSPN admits a PTAS. The result generalizes to other "fat" regions on the plane [25]. The algorithm can be modified to yield a PTAS for k-TSPN algorithm where the goal is to find the shortest tour which visits at least $k$ disks out of $n$ disks in the input. $k$-TSPN with uniform (intersecting) disks also admits a PTAS [23].

Elbassioni et al. presented a constant factor approximation algorithm for intersecting fat convex objects of comparable diameters where the tour is restricted to visit each object only at a finite set of specified points [16].

\subsection{Problem Definition}

Let $S=\left\{s_{1}, \ldots, s_{n}\right\}$ be a given set of the locations of $n$ sensors. For each sensor $s \in S$, define $D_{i n}$ as the inner disk (i.e. the disk centered at $s$ with radius $r_{i n}$ ) and $D_{\text {out }}$ as the outer disk (with radius $r_{\text {out }}$ ). The download time inside the inner disk is $T_{\text {in }}$ and the download time inside $D_{\text {out }}-D_{\text {in }}$ is $T_{\text {out }}$. Without loss of generality, we scale the distances so that the robot's maximum velocity is one unit.

The objective is to find a tour which visits either the inner disk or the outer disk of each sensor and minimizes the total time taken to travel and download data from all sensors. We refer to this problem as the Two-Ring Tour Problem (TRT). We also assume that the robot stops first and then downloads the data. Observe that the robot can reduce the total time of the tour by at most half if it downloads while travelling. Therefore our results yield approximation algorithms for simultaneous download model (increased by a factor 2) as well.

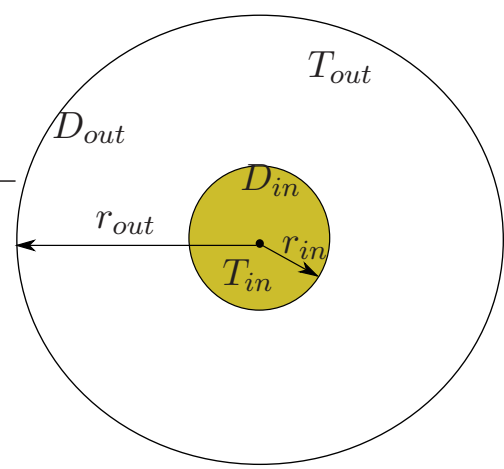

Figure 2: The two ring model. Download time is $T_{i n}$ in disk $D_{\text {in }}$ while it is $T_{\text {out }}$ in the region $D_{\text {out }}-D_{\text {in }}$.

The TRT problem is a generalization of the Eucledian TSP problem which is an NP-complete problem. This implies that the there is no algorithm which can solve the TRT problem in polynomial time unless $P=N P$. For such problems, approximation algorithms can be used to get an approximate solution. An approximation algorithm is an algorithm which runs in polynomial time on the size of input and guarantees that the solution is close to the optimal value. For minimization problems like TRT, an $\alpha$-approximation algorithm gives a solution which is at most $\alpha$ times of the optimal value regardless of the input instance. Before we present 
algorithms for TRT, we investigate some of the structural properties of TRT instances.

\section{Structural Properties}

In this section, we present some basic properties of an optimal TRT solution.

Proposition $1\left|C_{\text {in }}\right| \geq\left|C_{\text {out }}^{*}\right|$, where $\left|C_{\text {in }}\right|$ is any tour that visits all inner disks, and $C_{\text {out }}^{*}$ is the optimal TSPN tour visiting outer disks.

Proposition 1 follows from the fact that any tour that visits the inner disks also visits the outer disks. Using Proposition 1 we obtain a lower bound on the cost of the optimal solution to the TRT problem.

Proposition $2 O P T \geq\left|C_{\text {out }}^{*}\right|+n T_{\text {in }}$, where OPT is the cost incurred by the optimal TRT tour.

The first term on the right side of the inequality in Proposition 2 is the lower bound on the travel time taken by the optimal tour that visits all the sensors, while the second term is the lower bound on the time to download data from all the sensors.

The following result yields a lower bound on the travel component of the optimal solution. We will use this lower bound in some of the approximation algorithms presented later in this paper.

i

Lemma 1 For any three non-overlapping, equal-size disks on the plane, the length of any path that visits all three disks is lower bounded by $\alpha r$, where $r$ is the radius of the disks and $\alpha=0.4786$.

Proof. Let $\tau^{*}$ be the optimal TSPN solution visiting $n>2$ disks. Let $D_{1}, D_{2}$ and $D_{3}$ be three consecutive disjoint disks on $\tau^{*}$ with centers $c_{1}, c_{2}$ and $c_{3}$ respectively. Consider the segment of $\tau^{*}$ which visits these disks. $\tau^{*}$ either touches the boundary of a disk or it crosses the boundary twice. For each disk $D_{i}$, we identify a point $t_{i}$ which is either the touching point or one of the points where $\tau^{*}$ crosses $D_{i}$ as follows (see Figure 3):

- If $\tau^{*}$ touches $D_{2}$, pick the touching point as $t_{2}$, otherwise pick one of the two points on the boundary of $D_{2}$ arbitrarily which is crossed by $\tau^{*}$

- If $\tau^{*}$ touches $D_{1}$, pick the touching point as $t_{1}$, otherwise pick the closest crossing point to $t_{2}$ as $t_{1}$ (similarly choose $t_{3}$ )

We will present a series of transformations on the disk locations such that the length of $\tau^{*}$ will not increase. Afterwards, we will present a lower bound on $\left|t_{1} t_{2}\right|+\left|t_{2} t_{3}\right|$ in the transformed version which also applies to the original instance before the transformations.

In the first transformation, we will replace points $t_{1}$ and $t_{3}$. In the second transformation, we will move disks $D_{1}$ and $D_{3}$ in such a way that $D_{2}$ touches both disks. Both of these transformations will be done without increasing the total distance. Finally, we will establish the lower bound by optimizing the location of $t_{2}$.

Let $t_{1}^{\prime}$ be the point that segment $\left[t_{2} c_{1}\right]$ crosses the boundary of $D_{1}$. By moving $t_{1}$ to $t_{1}^{\prime}$, we do not increase the total distance (see Figure 3). Same observation can be applied for $t_{3}$ and $t_{3}^{\prime}$.

If either $\left|t_{1} t_{2}\right|$ or $\left|t_{2} t_{3}\right|$ is greater than or equal to $0.4786 r$, lower bound holds since the total distance is at least as claimed. If both distances are less than $0.4786 r$ then we do the following transformation. Without loss of generality, let us assume that $D_{1}$ is to the left of $D_{3}$ (see left of Figure 4). If $D_{1}$ touches $D_{2}$, we do not move $D_{1}$. Otherwise, we rotate $D_{1}$ along $t_{2}$ in counterclockwise direction until $D_{1}$ touches $D_{2}$. Similarly, if $D_{3}$ does not touch $D_{2}$, initially, we rotate it in clockwise direction until it touches $D_{2}$. Note that this transformation is only a rotation and does not change the total distance. This transformation is shown in Figure 4 (Middle).

In this transformed version of the problem, we formulate the total distance in terms of parameters $\theta=\angle t_{2} c_{2} c_{3}^{\prime}$ and $\beta=\angle c_{1}^{\prime} c_{2} c_{3}^{\prime}$ where $c_{1}^{\prime}$ and $c_{3}^{\prime}$ are centers of transformed disks 


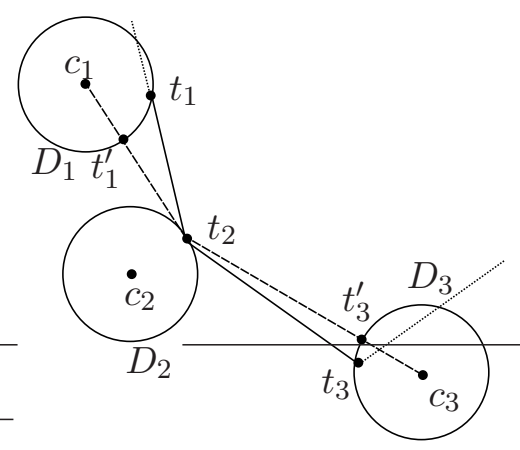

Figure 3: Three non-overlapping disks lying in a plane. The part of the optimal TSPN tour $t_{1}, t_{2}, t_{3}$ which visits disks $D_{1}, D_{2}$ and $D_{3}$, respectively. Without increasing the total distance, we can transform $t_{1}$ to $t_{1}^{\prime}$ and $t_{3}$ to $t_{3}^{\prime}$

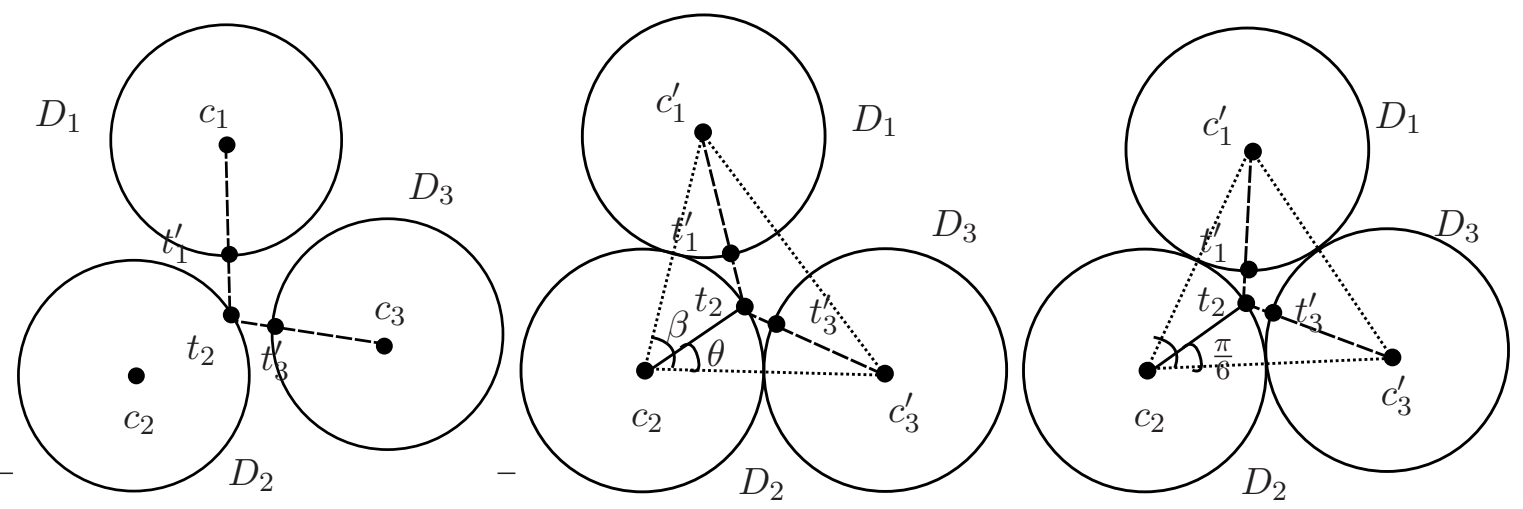

Figure 4: Left: Initial configurations of circles where $\left|t_{1}^{\prime} t_{2}\right|,\left|t_{2} t_{3}^{\prime}\right|<0.4786 r$. Middle: After rotation without changing the total distance, $D_{2}$ touches both $D_{1}$ and $D_{3}$. Right: The configuration where the total distance is minimum and equals to $0.4786 r$.

$D_{1}$ and $D_{3}$. Since $t_{2}$ is on the boundary of $D_{2}$, we can define all possible locations of $t_{2}$ in terms of $\theta$. Since $\left|t_{1} t_{2}\right|$ is less than $0.4786 r$, we can show that the angle $\angle c_{1}^{\prime} t_{2} c_{2}$ is greater than $\pi / 2$. Using the same fact on $\left|t_{2} t_{3}\right|$, we can show that the angle $\angle c_{3}^{\prime} t_{2} c_{2}$ is greater than $\pi / 2$. Together with the previous inequality, we establish that $t_{2}$ is inside the triangle $\triangle c_{1}^{\prime} c_{2} c_{3}^{\prime}$. Using the same distance constraints, we can show that $\beta$ is upper bounded by $\pi / 2$. Moreover, since all disks are non-overlapping, it is lower bounded by $\pi / 3$ (the configuration when all disks touch each other). The total distance can be expressed as:

$$
f(\theta, \beta)=r(\sqrt{5-4 \cos (\theta)}+\sqrt{5-4 \cos (\beta-\theta)}-2)
$$

To minimize Equation 1, we first solve for $\partial f / \partial \theta=0$. This yields $\theta=\beta / 2$. The corresponding minimum value is $2 r(\sqrt{5-4 \cos (\beta / 2)}-1)$. The value $f(\beta / 2, \beta)$ over the interval $\frac{\pi}{3} \leq \beta<\frac{\pi}{2}$ is minimized when $\beta=\pi / 3$. These two values yield a configuration where all the circles touch each other and $t_{2}$ is in the middle of tangent points. This configuration is shown in right of Figure 4. In this configuration the total distance is $0.4786 r$ which will be used in Theorem 1 to find a lower bound on the tour length.

We use Lemma 1 to find a lower bound on any tour of non-overlapping, equal-sized disks in a plane. This lower bound is used for analysis of algorithms presented in subsequent sections.

Theorem 1 Any tour $\tau$ of $n$ disjoint, equal-sized disks of radius $r$, satisfies

$$
|\tau| \geq \frac{n}{2} \alpha r
$$


where $\alpha=0.4786$ and $n \geq 3$.

Proof. Take the tour $\tau$. It will give an order of sensors in which to visit them. Let the order be $s_{1}, s_{2}, s_{3}, \ldots, s_{n}$. From Lemma 1 we know that the cost of every sub-path $P_{i}$ which joins $s_{i}, s_{i+1}$ and $s_{i+2}$ in $\tau$, is lower bounded by $\alpha r$ for every $i \in 1, n$. Also $|\tau| \geq \frac{1}{2} \sum_{i=1}^{n}\left|P_{i}\right|$. Therefore $|\tau| \geq \frac{n}{2} \alpha r$.

\section{The General Case}

In the general case, the communication disks of sensors are placed arbitrarily on the plane possibly overlapping. This section presents three algorithms for this case.

Our first algorithm uses algorithms for TSPN and k-TSPN problems as subroutines. If TSPN solution is $p$-approximate and k-TSPN solution is $q$-approximate then our algorithm gives a $(p+q)$-approximate solution for the TRT problem. For example, we can use the PTAS for k-TSPN $[23,25]$ and PTAS for TSPN from [13] to get a $(2+\epsilon)$-approximate solution for TRT (where $\epsilon$ can be made arbitrarily small at the expense of running time).

PTAS algorithms are generally difficult to implement and they have high running times in practice. Therefore, we present two algorithms which are easy to implement and have approximation ratios of $O\left(\frac{T_{\text {out }}}{T_{\text {in }}}\right)$ and $O\left(\frac{r_{\text {out }}}{r_{\text {in }}}\right)$ where $T_{\text {out }}>T_{\text {in }}>0$. In practice, the ratios $\frac{T_{\text {out }}}{T_{\text {in }}}$ and $\frac{r_{\text {out }}}{r_{\text {in }}}$ are expected to be small, therefore the two algorithms are relevant for the real world instances of TRT.

\subsection{General Approximation Algorithm}

Let $C^{*}$ be the optimal tour, $S_{I}$ be the set of sensors whose inner disks are visited by $C^{*}$, and $S_{O}$ be the remaining sensors whose outer disks are visited by $C^{*}$. We have $\left|S_{I}\right|+\left|S_{O}\right|=n$. The total cost of this tour is $O P T=\left|C^{*}\right|+\left|S_{I}\right| T_{\text {in }}+\left|S_{O}\right| T_{\text {out }}$ where $T_{\text {in }}$ and $T_{\text {out }}$ are the download times from the inner and outer disks of a single sensor.

Let $f(k)$ be the cost incurred by an algorithm $A$ which computes a $k$-TSPN tour of inner disks using a $q$-approximation algorithm and visits these sensors. Afterwards, $A$ downloads data from the remaining sensors by visiting their outer disk. We claim that the cost incurred by $A$ for $k=\left|S_{I}\right|$ is a $(p+q)$-approximation. In other words, $f\left(\left|S_{I}\right|\right) \leq(p+q) O P T$.

To see this, first observe that $C^{*}$ is a $k$-TSPN tour (with $k=\left|S_{I}\right|$ ) of the inner disks and therefore, the optimal $k$-TSPN tour of all the inner disks is no longer than $C^{*}$. Let $C_{1}^{*}$ be the optimal $k$-TSPN tour. By the previous argument, $\left|C_{1}^{*}\right| \leq\left|C^{*}\right|$. Since we are using a $q$ approximation algorithm for $k$-TSPN, the tour $C_{1}$ given by this algorithm will be of length at most $q\left|C_{1}^{*}\right|$.

Next, let $C_{2}^{*}$ be an optimal TSPN tour of the outer disks of sensors not visited by $C_{1}$. This tour will be shorter or equal in length than the optimal tour $C_{\text {out }}^{*}$ which visits all the outer disks, i.e., $\left|C_{2}^{*}\right| \leq\left|C_{\text {out }}^{*}\right|$. But the length of the optimal tour of the outer disks is a lower bound on the length $C^{*}$ (Proposition 1). Therefore, $\left|C_{\text {out }}^{*}\right| \leq\left|C^{*}\right|$. We compute a $p$-approximate TSPN tour by using a $p$-approximation algorithm for the TSPN problem. If $C_{2}$ is the tour given by this algorithm, then we have $\left|C_{2}\right| \leq p\left|C^{*}\right|$

Therefore, the total cost incurred by $\left|C_{1} \cup C_{2}\right|$ is

$$
\begin{aligned}
f\left(\left|S_{I}\right|\right) & =\left|C_{1}\right|+\left|C_{2}\right|+\left|S_{I}\right| T_{\text {in }}+\left|S_{O}\right| T_{\text {out }} \\
& \leq q\left|C^{*}\right|+p\left|C^{*}\right|+\left|S_{I}\right| T_{\text {in }}+\left|S_{O}\right| T_{\text {out }} \\
& \leq(p+q) O P T
\end{aligned}
$$

Finally, we observe that $\min _{k} f(k) \leq f\left(\left|S_{I}\right|\right)$. Therefore, by picking the value of $k$ which minimizes $f(k)$ provides a $(p+q)$-approximation. Algorithm 1 implements the steps mentioned above. It iterates over all possible values of $k$ (from 1 to $n$ ) and picks the one for which the total cost is minimized. 


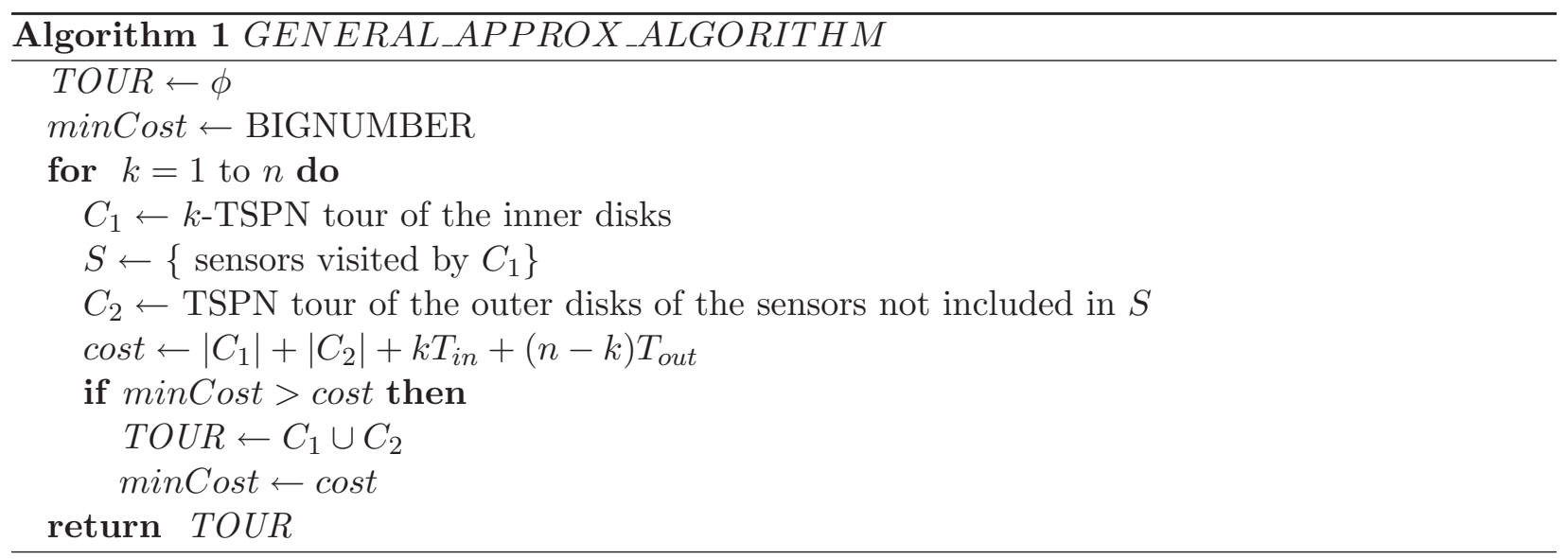

Mitchell presents PTAS algorithms for both TSPN and k-TSPN problems [25]. If we use these algorithms for the case when the outer disks are disjoint, then we get $p=(1+\epsilon / 2)$ and $q=(1+\epsilon / 2)$. In that case our algorithm yields a $(2+\epsilon)$-approximation factor. In the remaining case (intersecting outer disks), we can use the constant factor approximation algorithm for the outer disks $[13]$ to find a TSPN tour $(p=11.5)$ and the the PTAS for inner disks which yields an approximation algorithm with factor $(12.15+\epsilon)$ for TRT.

\section{$3.2 O\left(\frac{T_{o u t}}{T_{\text {in }}}\right)$-approximation}

The algorithm presented in this section is appropriate for the case when the download times of the inner and the outer disks are comparable. For this scenario, we show that the strategy of visiting just the outer disks of the sensors yields an $O\left(\frac{T_{\text {out }}}{T_{\text {in }}}\right)$-approximation for TRT.

First, we use a TSPN algorithm (e.g. [13]) to find a TSPN tour $C_{\text {out }}$ of all the outer disks. Let $p$ be the approximation factor for this algorithm and $p \geq 1$. Since the tour visits the outer disks, the robot downloads data from the sensors with the download speed of $T_{\text {out }}$. Therefore, the approximation factor of the algorithm is:

$$
\begin{aligned}
\frac{\left|C_{\text {out }}\right|+n T_{\text {out }}}{O P T} & \leq \frac{\left|C_{\text {out }}\right|+n T_{\text {out }}}{\left|C_{\text {out }}^{*}\right|+n T_{\text {in }}} \\
& \leq \frac{p\left|C_{\text {out }}^{*}\right|+n T_{\text {out }}}{\left|C_{\text {out }}^{*}\right|+n T_{\text {in }}} \\
& \leq p \frac{\left|C_{\text {out }}^{*}\right|+n T_{\text {out }}}{\left|C_{\text {out }}^{*}\right|+n T_{\text {in }}} \leq p \frac{T_{\text {out }}}{T_{\text {in }}}
\end{aligned}
$$

The first inequality in Equation 3 comes from Proposition 2. TSPN approximation directly yields the second inequality. Therefore, our algorithm has an approximation factor of $O\left(\frac{T_{\text {out }}}{T_{\text {in }}}\right)$.

\section{3 $O\left(\frac{r_{\text {out }}}{r_{\text {in }}}\right)$-approximation}

For some problem instances the ratio of radii may be better than the ratio of download times. In such cases if the ratio of radii is small, we can find an efficient algorithm with the approximation factor of order $O\left(\frac{r_{\text {out }}}{r_{\text {in }}}\right)$.

In this algorithm, first we compute a maximal non-overlapping set $I$ of the outer disks. For this we use Algorithm 2. Then we find a TSPN tour $C_{\text {out }}^{I}$ of the disks in $I$. For each disk $A \in I$, we define the set of sensors whose outer disks intersect with $A$ as $S_{A}$. Next, we will show how we can extend $C_{\text {out }}^{I}$ such that it visits all the inner disks of the sensors in $S_{A}$. In the following, we assume that $|I| \geq 3$. 
Let $D$ be a disk of radius $2 r_{\text {out }}$ and is co-centered with $A$. All the sensors in $S_{A}$ lie on or in $D$. We traverse $D$ in concentric circles which are distance $r_{i n}$ apart as shown in Figure 5 . This will ensure that all the inner disks of the sensors in $S_{A}$ are visited.
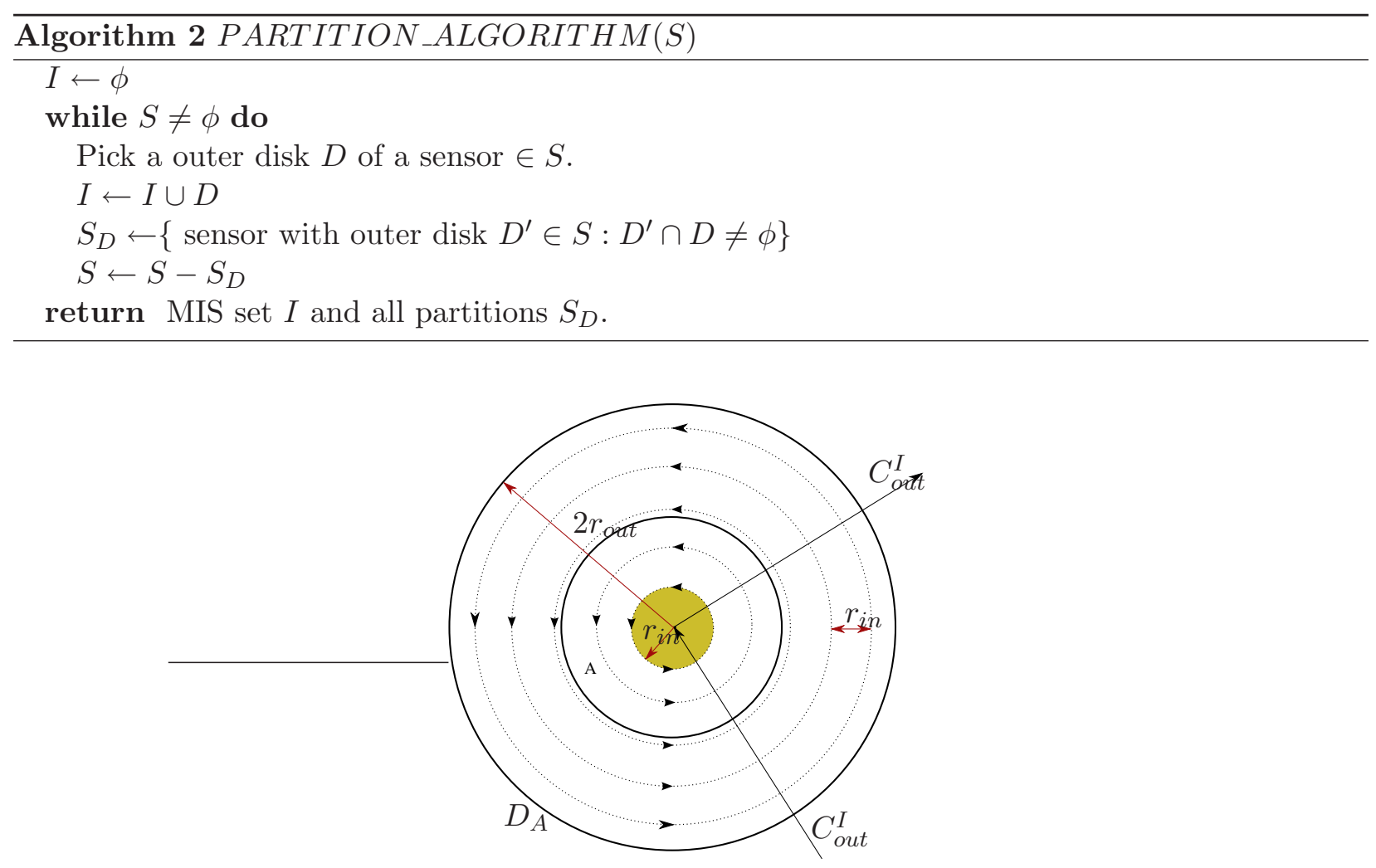

Figure 5: In all inner-disk visits, the algorithm chooses to sweep the $2 r_{\text {out }}$ size disk centered at $A$ in concentric circles which are $r_{i n}$ apart.

Let $d_{A}$ be the extra distance traveled in this process and let $k=\left\lfloor\frac{2 r_{\text {out }}}{r_{\text {in }}}\right\rfloor$. Then, $d_{A}=$ $\sum_{i=1}^{k} 2 \pi i r_{i n}=\pi r_{\text {in }} k(k+1)=2 \pi r_{\text {out }}(k+1)$. The cost of this tour is $\left|C_{\text {out }}^{I}\right|+m d_{A}=\left|C_{\text {out }}^{I}\right|+$ $2 m \pi r_{\text {out }}(k+1)$, where $m=|I|$. This gives us the approximation ratio

$$
\begin{aligned}
\frac{\left|C_{\text {out }}^{I}\right|+m d_{A}+n T_{\text {in }}}{\left|C_{\text {out }}{ }^{*}\right|+n T_{\text {in }}} & \leq \frac{\left|C_{\text {out }}^{I}\right|+m d_{A}}{\left|C_{\text {out }}{ }^{*}\right|} \\
& =\frac{\left|C_{\text {out }}^{I}\right|}{\left|C_{\text {out }}{ }^{*}\right|}+\frac{2 m \pi r_{\text {out }}(k+1)}{\left|C_{\text {out }}^{*}\right|} \\
& \leq p+\frac{2 m \pi r_{\text {out }}(k+1)}{\frac{m}{2} r_{\text {out }} \alpha} \\
& =p+\frac{4 \pi(k+1)}{\alpha}
\end{aligned}
$$

In the second inequality we use $p$ as the approximation ratio for the TSPN algorithm and the lower bound on $C_{\text {out }}^{*}$ is obtained from Theorem 1. Finally, this gives us an $O\left(\frac{r_{\text {out }}}{r_{\text {in }}}\right)$ approximation algorithm under the requirement that $|I| \geq 3$.

\section{Special Cases}

In this section, we consider efficient solutions for some practical sensor deployments. In the first scenario, we consider a sparse network deployment where communication disks do not overlap. 
In the second scenario, we consider a common network topology where the sensors are deployed uniformly over a grid.

\subsection{Non-Overlapping Outer Disks}

In this section we consider the case when all the outer disks are non-overlapping. Our algorithm is simple. We compute a TSPN tour $C_{i n}$ of inner disks and download data from inner disks with cost of $T_{i n}$ at each disk. This tour can be computed by visiting centers of the disks in polynomial time with $(1+\epsilon)$ approximation using PTAS given in [13].

When $n=1$, the solution is trivial. For $n=2$, all possible cases (visiting both inner disks, both outer disks or one inner and one outer disk) can be calculated and the one giving the minimum cost is picked. For $n \geq 3$ we present the following lemma.

Lemma 2 Given $n$ equal size disk of radius $r$, where $n \geq 3$, one can compute a TSPN tour $C_{\text {in }}$ of the inner disks such that

$$
\frac{\left|C_{i n}\right|+n T_{i n}}{\left|C_{\text {out }}^{*}\right|+n T_{i n}} \leq(1+\epsilon)\left(1+\frac{4}{\alpha}\right)
$$

Proof. We observe that any outer disk TSPN tour can be converted in to an inner disk TSPN tour by extending it at most $2\left(r_{o}-r_{i}\right)$ in length at each disk. From this observation we get

$$
\left|C_{\text {in }}^{*}\right| \leq\left|C_{\text {out }}^{*}\right|+2 n\left(r_{o}-r_{i}\right) \leq\left|C_{\text {out }}^{*}\right|+2 n r_{o},
$$

where $C_{i n}^{*}$ is the optimal inner disk TSPN tour. Therefore,

$$
\begin{aligned}
\frac{\left|C_{\text {in }}\right|+n T_{\text {in }}}{\left|C_{\text {out }}^{*}\right|+n T_{\text {in }}} & \leq(1+\epsilon) \frac{\left|C_{\text {in }}^{*}\right|}{\left|C_{\text {out }}^{*}\right|} \leq(1+\epsilon)\left(1+\frac{2 n r_{o}}{\left|C_{\text {out }}^{*}\right|}\right) \\
& \leq(1+\epsilon)\left(1+\frac{4}{\alpha}\right)
\end{aligned}
$$

Equation 7 is obtained by applying the lower bound obtained in Theorem 1 to Equation 6 . The $(1+\epsilon)$-approximation is obtained by the PTAS for finding TSPN tour of non-overlapping equal size disks given in [13].

This gives us the following result.

Theorem 2 A TSPN tour of inner disks is a factor $\left(1+\frac{4}{\alpha}\right)$-approximation for TRT with nonoverlapping outer disks $(\alpha=0.4786)$.

\subsection{Grid Based Deployment}

In this section, we consider a common scenario where $n^{2}$ sensors are deployed on the vertices of an $n \times n$ grid. Let us define a boustrophedon path as a path that goes back and forth along a fixed direction (vertical or horizontal) until it touches the boundary. For the case of grid-based deployment, there exists an optimal solution OPT which follows a boustrophedon path. In other words, assuming OPT starts from the top left corner of the grid, first it moves right until it reaches the first vertical line, then follows the vertical line downwards until the bottom of the grid. It then moves right to a vertical line and follows it upwards until it reaches the top and so on. Therefore if we compute the set of vertical lines traversed by OPT, we can construct the TRT path. Note that this path does not necessarily go through each sensor location (centers of the disks), it simply intersects one of the disks of each sensor.

We restrict the candidate vertical lines to the set of tangent lines $L$. Each sensor introduces four vertical tangent lines: two tangent to the outer disk and two tangent to the inner disk. It is easy to show that if there exists a solution where there is a vertical line in between two tangent lines in $L$ then we can replace this line with one of its neighbor tangent lines to achieve the same cost. Figure 6 shows an instance (only one row) and its vertical tangent lines. From now on, we focus on selecting a subset of $L$ such that the total time is minimized. 


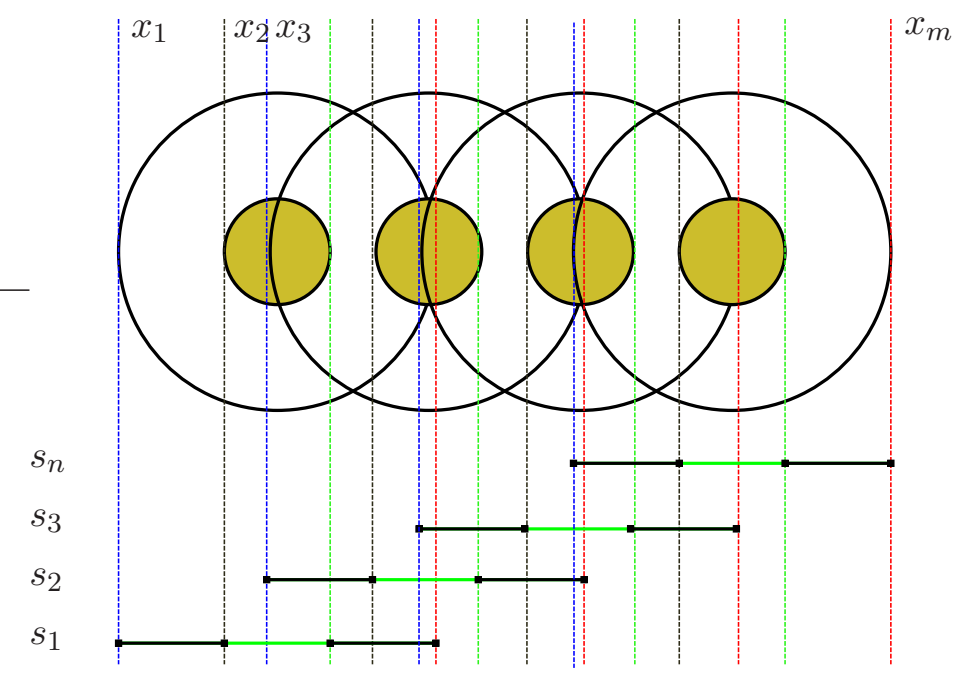

Figure 6: Sensors are arranged on an $n \times n$ grid. For each sensor, draw vertical tangents. Two for the outer disk and two for the inner disk. The number of tangents $=m \leq 4 n$. The stabbing lines are chosen from this set of tangents.

\section{LP-formulation}

For each column $i$ where $1 \leq i \leq n$, we define four binary variables: $x_{4 i-3}$ for the left outer tangent line, $x_{4 i-2}$ for the left inner tangent line, $x_{4 i-1}$ for the right inner tangent line and $x_{4 i}$ for the right outer tangent line. We set $x_{j}=1$ if and only if the tangent line $x_{j}$ is traversed. We define a variable $y_{i}$ such that $y_{i}=1$ iff the robot visits the inner disks of sensor column $i$.

For each column at least one tangent line should be visited hence we have (i) $x_{4 i-3}+x_{4 i-2}+$ $x_{4 i-1}+x_{4 i} \geq 1$. Moreover, if one of the inner tangent lines is visited then $y_{i}$ should be set to 1 . We satisfy this by the following two constraints: (ii) $y_{i} \leq x_{4 i-2}+x_{4 i-1}$ and (iii) $y_{i} \leq 1$.

Finally we define the cost of the solution. Let $C$ be the cost of traveling a stabbing line, $T_{i n}$ (resp. $T_{\text {out }}$ ) be the cost of downloading from inner (resp. outer) disks from an entire column.

The total download time is

$$
C \sum_{j=1}^{4 n} x_{j}+\left(T_{\text {in }}-T_{\text {out }}\right) \sum_{i=1}^{n} y_{i}+n T_{\text {out }}
$$

The integer solution to the above cost function under constraints (i)-(iii) gives us the optimal solution for the uniform case. The complete integer linear program solution is given below:

minimize

$$
C \sum_{j=1}^{4 n} x_{j}+\left(T_{\text {in }}-T_{\text {out }}\right) \sum_{i=1}^{n} y_{i}+n T_{\text {out }}
$$

such that

$$
\begin{array}{ll}
x_{4 i-3}+x_{4 i-2}+x_{4 i-1}+x_{4 i} \geq 1 & 1 \leq i \leq n \\
y_{i} \leq x_{4 i-2}+x_{4 i-1} & 1 \leq i \leq n \\
y_{i} \leq 1 & 1 \leq i \leq n \\
x_{i}, y_{i} \in\{0,1\} &
\end{array}
$$

Next we show that the relaxed version of this ILP can be rounded to obtain a 4-approximation. 


\section{Relaxation and Rounding}

We relax $x_{j}$ and $y_{i}$ by replacing the binary constraints with $x_{j} \geq 0$ and $y_{i} \geq 0$. Let $O P T(L P)$ be the cost incurred by the optimal solution to the relaxed version. After solving the LP relaxation, we round the solution as follows: If $x_{j} \geq \frac{1}{4}$ we set it to one. Otherwise, we set it to zero. These are the vertical lines that will be traversed. We then use the values of $x_{j}$ to determine the values of $y_{i}$. Let $S O L$ be the cost incurred by this integer solution, and $O P T(I L P)$ be the cost incurred by the optimal binary solution. Observe that $O P T(L P) \leq O P T(I L P)$ and $S O L \leq 4 O P T(L P)$. Therefore $S O L \leq 4 O P T(I L P)$.

We now show that the rounding gives a feasible solution. To see this, observe that due to constraint (i), one of $x_{4 i-3}, x_{4 i-2}, x_{4 i-1}$ and $x_{4 i}$ is at least $\frac{1}{4}$. Therefore, after rounding at least one of these stabbing lines is selected, which means that data from every sensor column is collected.

\section{Experiments}

We performed experiments using wireless sensors (telosB motes) and a custom-built robot. First, we obtained the model parameters. Figure 7 shows the average download times as a function of distance from the sensor. For each distance value $d$, we moved the base mote on a disc centered at the sensor mote location with radius $d$. In each trial, we downloaded 100 packets and recorded their download times. The blue line in the figure shows the average download times and the red error bar shows the minimum and maximum download times observed during each trial. The download times show us that until a distance of 30 feet (gray line) the communication between sensor and base motes is reliable and the download times are short. However beyond 30 feet the communication becomes very unreliable: long download times up to as much as 22 seconds were observed. Beyond 45 feet there was no communication.

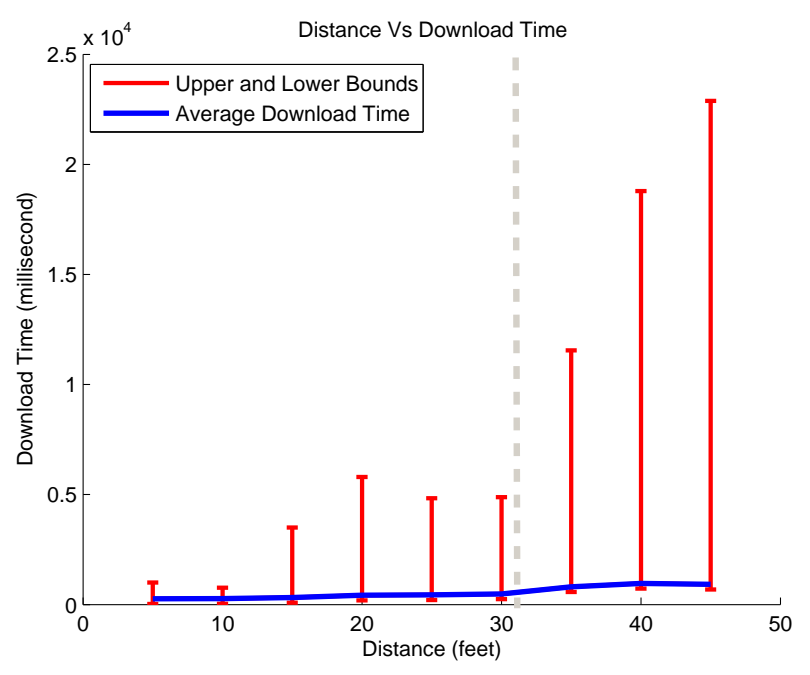

Figure 7: Download speed vs distance for communication between two sensor motes.

Next, we conducted real experiments with a robot to compare the performance of various TRT tours. We used an outdoor robot developed in our lab to collect data from four telosB sensors. These sensors were deployed on the corners of a square field of size 70x70 feet (See Figure 8). The inner disk radius was set to 18 feet and the outer disk radius was chosen 30 feet.

We tested three natural strategies and compared their performances. In the first strategy the robot followed a TSP tour which visits the centers. In the second and third strategies, the robot visited the outer and inner disks respectively. In each visit the robot downloaded 100 packets and the time to download varied according to the packet loss. 


\begin{tabular}{|l||l|l|l|}
\hline & Download & Travel & Total \\
\hline \hline Visit Disk Centers & 8 & 140 & 148 \\
Visit Outer Disks & 50 & 64 & 114 \\
Visit Inner Disks & 9 & 119 & 128 \\
\hline
\end{tabular}

Table 1: Table showing the download and travel times from the three strategies.

First we treated the TRT problem as a TSP problem and made the robot visit the sensor locations. In this case the total download time was 8 seconds where as the travel time was 140 seconds (see Figure 9 and Table 1). In the second experiment, the robot visited the outer disks which dropped the tour time from 148 seconds to 114 seconds. In this case the download time and the travel time were 50 seconds and 64 seconds respectively. In the last experiment, the robot followed an inner disk tour. The download time drastically decreased from 50 seconds to 9 seconds in this case. However, the travel time increased from 64 seconds to 119 seconds and the overall tour time increased by 14 seconds to 128 seconds.

The experiment demonstrates that the wireless range can be exploited to reduce data gathering time, and paying attention to the inner and outer disk parameters can yield further savings.
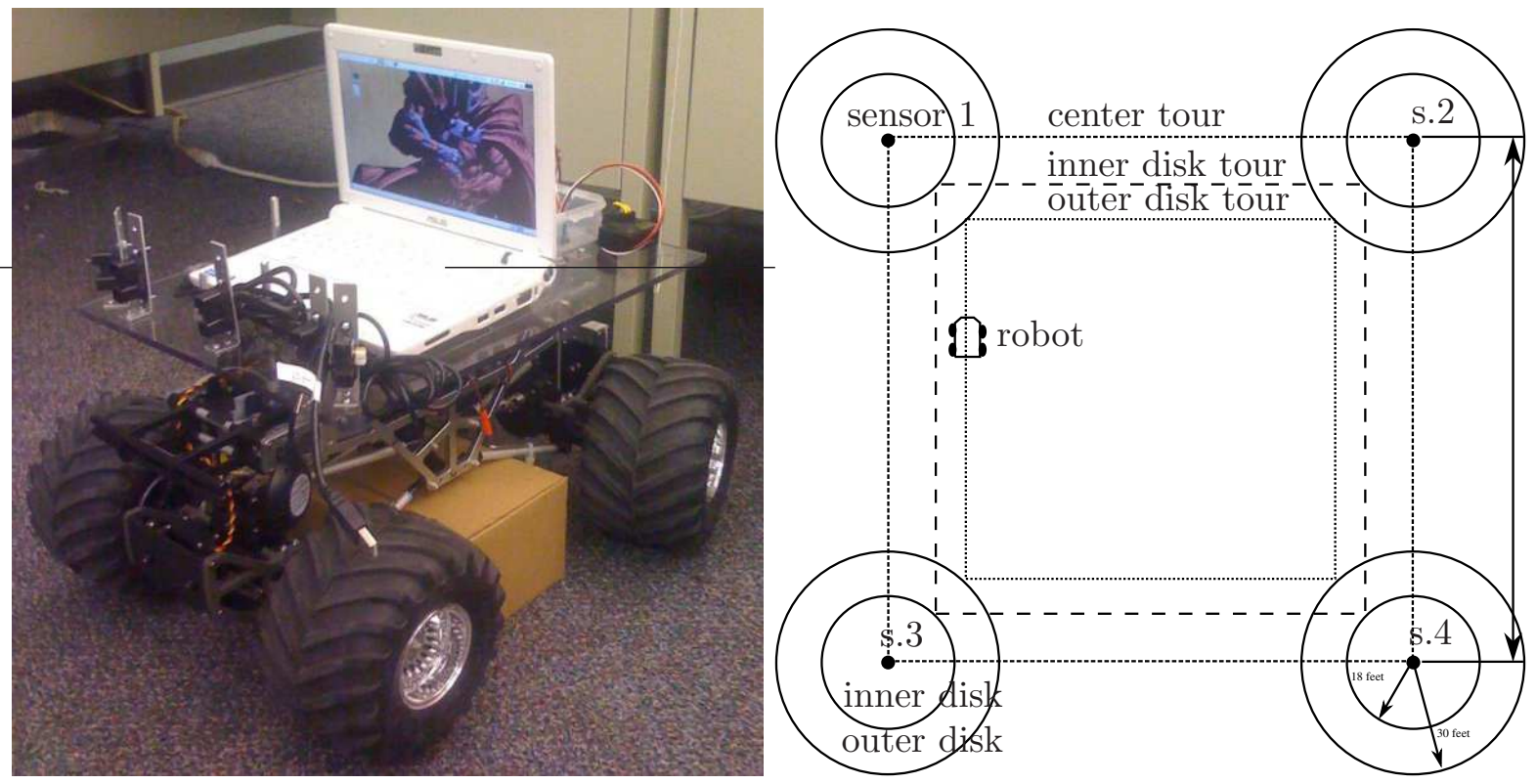

70 feet

Figure 8: Left: An outdoor robot developed in our lab was used in the experiments for validating the two-ring model. Right: The setup of the experiment.

\section{Concluding Remarks}

In this paper, we introduced a novel robot routing problem that arises in wireless applications where a mobile entity is charged with downloading data from a set of nodes in the least amount of time. The novelty of the formulation lies in the way download time is modeled: two concentric disks around each sensor with different download times. The mobile entity must decide which disk to visit for each sensor, and spend the corresponding time to download its data.

For the general case, we first presented an algorithm whose approximation is a function of the approximations to k-TSPN and TSPN problems. We also presented two polynomial time algorithms whose performance ratios are proportional to the ratio of the two radii or the ratio 

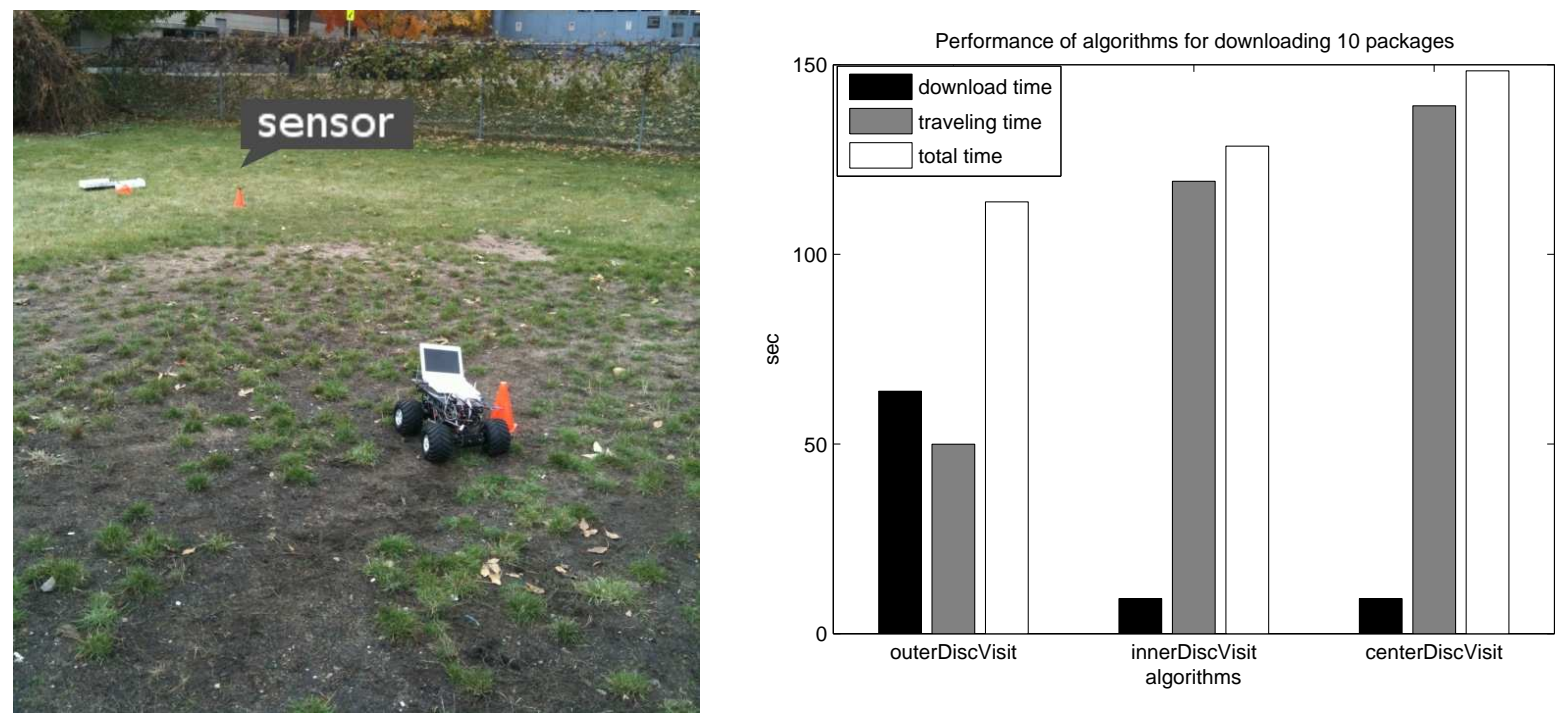

Figure 9: Left: A snapshot from the experiment. Right: Download and travelling time of the following strategies: visit outer disk, visit inner disks and visit disk centers.

of the download times. As demonstrated in an experimental setting, these two parameters are usually small in some practical scenarios.

For two special but common cases of the problem (uniform deployment and sparse deployments where the outer disks are disjoint), we presented constant factor approximation algorithms.

Generalization of TRT problem to polygonal environments possibly with obstacles remains an open research problem. If the obstacles do not intersect with the communication disks, some of the algorithms in this paper can be extended to solve this case (in particular the algorithms in Sections 3.2 and 3.3). When obstacles intersect with the communication disks, the problem becomes even harder. Our future work includes solving the TRT problem in complex environments, and extensions to multiple robots.

\section{References}

[1] K. Akkaya and M. Younis. A survey on routing protocols for wireless sensor networks. Ad Hoc Networks, 3(3):325 - 349, 2005.

[2] G. Anastasi, M. Conti, and M. Di Francesco. Data collection in sensor networks with data mules: An integrated simulation analysis. In IEEE Symposium on Computers and Communications, pages 1096 -1102, Marrakech,Morocco, 2008.

[3] G. Anastasi, M. Conti, E. Monaldi, and A. Passarella. An adaptive data-transfer protocol for sensor networks with Data Mules. In IEEE International Symposium on a World of Wireless, Mobile and Multimedia Networks, pages 1-8, Helsinki, Finland, 2007.

[4] E. M. Arkin and R. Hassin. Approximation algorithms for the geometric covering salesman problem. Discrete Applied Mathematics, 55:197-218, 1995.

[5] S. Arora. Polynomial time approximation schemes for euclidean traveling salesman and other geometric problems. J. ACM, 45(5):753-782, 1998.

[6] A. Beaufour, M. Leopold, and P. Bonnet. Smart-tag based data dissemination. In Proceedings of the 1st ACM International Workshop on Wireless Sensor Networks and Applications, pages 68-77, Atlanta, GA, USA, 2002. ACM.

[7] D. Bhadauria, O. Tekdas, and V. Isler. Robotic data mules for collecting data over sparse sensor fields. Journal of Field Robotics, 28(3):388-404, 2011. 
[8] L. Boloni and D. Turgut. Should I send now or send later? A decision-theoretic approach to transmission scheduling in sensor networks with mobile sinks. Wireless Communications and Mobile Computing, 8(3):385-403, 2008.

[9] A. Chakrabarti, A. Sabharwal, and B. Aazhang. Using predictable observer mobility for power efficient design of sensor networks. In Proceedings of the 2nd International Conference on Information Processing in Sensor Networks, pages 129-145, Palo Alto, CA, USA, 2003. Springer-Verlag.

[10] Y. Chen and A. Terzis. Poster abstract: On the spatial characteristics of the gray region for 802.15.4 radios. In Information Processing in Sensor Networks, 2009. IPSN 2009. International Conference on, pages 393 -394, 2009.

[11] N. Christofides. Worst-case analysis of a new heuristic for the travelling salesman problem. Technical Report 388, Carnegie-Mellon University, 1976.

[12] S. Diggavi, M. Grossglauser, and D. Tse. Even one-dimensional mobility increases ad hoc wireless capacity. In 2002 IEEE International Symposium on Information Theory, 2002. Proceedings, page 352, 2002.

[13] A. Dumitrescu and J. S. B. Mitchell. Approximation algorithms for tsp with neighborhoods in the plane. J. Algorithms, 48(1):135-159, 2003.

[14] M. Dunbabin, P. Corke, I. Vasilescu, and D. Rus. Data muling over underwater wireless sensor networks using an autonomous underwater vehicle. In IEEE International Conference on Robotics and Automation, pages 2091-2098, Orlando, FL, USA, 2006.

[15] E. Ekici, Y. Gu, and D. Bozdag. Mobility-based communication in wireless sensor networks. IEEE Communications Magazine, 44(7):56, 2006.

[16] K. Elbassioni, A. V. Fishkin, and R. Sitters. On approximating the tsp with intersecting neighborhoods. In Proc. 17th Annu. Internat. Sympos. Algorithms Comput, 2006.

[17] Y. Gu, D. Bozdag, E. Ekici, F. Ozguner, and C. Lee. Partitioning based mobile element scheduling in wireless sensor networks. In Second Annual IEEE Communications Society Conference on Sensor and Ad Hoc Communications and Networks, pages 386-395, Santa Clara, CA, USA, 2005.

[18] D. Jea, A. Somasundara, and M. Srivastava. Multiple controlled mobile elements (data mules) for data collection in sensor networks. In International Conference on Distributed Computing in Sensor Systems, pages 244-257, Marina del Rey, CA, USA, 2005. Springer.

[19] P. Juang, H. Oki, Y. Wang, M. Martonosi, L. Peh, and D. Rubenstein. Energy-efficient computing for wildlife tracking: Design tradeoffs and early experiences with zebranet. $A C M$ SIGOPS operating systems review, 36(5):96-107, 2002.

[20] A. Kansal, A. A. Somasundara, D. D. Jea, M. B. Srivastava, and D. Estrin. Intelligent fluid infrastructure for embedded networks. In International Conference on Mobile Systems, Applications, and Services, pages 111-124, Boston, MA, USA, 2004.

[21] J. Luo and J.-P. Hubaux. Joint mobility and routing for lifetime elongation in wireless sensor networks. In INFOCOM 2005. 24th Annual Joint Conference of the IEEE Computer and Communications Societies. Proceedings IEEE, volume 3, pages 1735 - 1746 vol. 3, march 2005.

[22] S. Meguerdichian, F. Koushanfar, M. Potkonjak, and M. B. Srivastava. Coverage problems in wireless ad-hoc sensor networks. In IEEE INFOCOM, pages 1380-1387, 2001.

[23] J. S. B. Mitchell. Personal Communication.

[24] J. S. B. Mitchell. Guillotine subdivisions approximate polygonal subdivisions: A simple polynomial-time approximation scheme for geometric tsp, k-mst, and related problems. SIAM J. Comput., 28(4):1298-1309, 1999.

[25] J. S. B. Mitchell. A ptas for tsp with neighborhoods among fat regions in the plane. In SODA '07: Proceedings of the eighteenth annual ACM-SIAM symposium on Discrete algorithms, pages 11-18, Philadelphia, PA, USA, 2007. Society for Industrial and Applied Mathematics. 
[26] Vineyard uses sensor network to fine-tune irrigation. NetworkWorld, 2009.

[27] R. Shah, S. Roy, S. Jain, and W. Brunette. Data mules: Modeling and analysis of a three-tier architecture for sparse sensor networks. Ad Hoc Networks, 1(2-3):215-233, 2003.

[28] R. Sugihara and R. Gupta. Optimal Speed Control of Mobile Node for Data Collection in Sensor Networks. IEEE Transactions on Mobile Computing, pages 127-139, 2009.

[29] O. Tekdas, J. Lim, A. Terzis, and V. Isler. Using mobile robots to harvest data from sensor fields. IEEE Wireless Communications, 2008. Special Issue on Wireless Communications in Networked Robotics.

[30] Y. Tirta, Z. Li, Y.-H. Lu, and S. Bagchi. Efficient collection of sensor data in remote fields using mobile collectors. In International Conference on Computer Communications and Networks, pages 515-519, Chicago, IL, USA, 2004.

[31] W. Wang, V. Srinivasan, and K. Chua. Using mobile relays to prolong the lifetime of wireless sensor networks. In Proceedings of the 11th annual international conference on Mobile computing and networking, page 283. ACM, 2005.

[32] F. Wu, C. Huang, and Y. Tseng. Data gathering by mobile mules in a spatially separated wireless sensor network. In International Conference on Mobile Data Management: Systems, Services and Middleware, pages 293-298, Taipei,Taiwan, 2009. IEEE Computer Society.

[33] J. Xing, H. Wang, K. Han, D. Ray, C. Huang, L. Chemnick, C. Stewart, T. Disotell, O. Ryder, and M. Batzer. A mobile element based phylogeny of Old World monkeys. Molecular Phylogenetics and Rvolution, 37(3):872-880, 2005.

[34] B. Yuan, M. Orlowska, and S. Sadiq. On the optimal robot routing problem in wireless sensor networks. IEEE Trans. on Knowl. and Data Eng., 19(9):1252-1261, 2007.

[35] J. Zhao and R. Govindan. Understanding packet delivery performance in dense wireless sensor networks. In Proceedings of the 1st international conference on Embedded networked sensor systems, pages 1-13. ACM, 2003.

[36] W. Zhao, M. Ammar, and E. Zegura. A message ferrying approach for data delivery in sparse mobile ad hoc networks. In Proceedings of the 5th ACM international symposium on Mobile ad hoc networking and computing, pages 187-198. ACM, 2004. 